\title{
Educação em dor em pacientes com lombalgia e sensibilização central: um estudo
}

\author{
piloto \\ Pain education in patients with low back pain and central sensitization: a pillot study \\ Educación sobre el dolor en pacientes con lumbalgia y sensibilización central: un estudio piloto
}

Recebido: 23/08/2021 | Revisado: 30/08/2021 | Aceito: 02/09/2021 | Publicado: 05/09/2021

\author{
Viviane Nascimento Brandão Lima \\ ORCID: https://orcid.org/0000-0003-4650-2391 \\ Universidade Federal de Sergipe, Brasil \\ E-mail: vivianenbl@gmail.com \\ Ana Verena Calmon Almeida \\ ORCID: https://orcid.org/0000-0001-8579-0045 \\ Universidade Federal de Sergipe, Brasil \\ E-mail: calmonverena@gmail.com \\ Gessica Uruga Oliveira \\ ORCID: https://orcid.org/0000-0002-3960-6742 \\ Universidade Federal de Sergipe, Brasil \\ E-mail: gessicauruga@gmail.com \\ Victor Augusto Barreto Monteiro \\ ORCID: https://orcid.org/0000-0002-2365-7422 \\ Universidade Federal de Sergipe, Brasil \\ E-mail: victoraugusto36@live.com \\ Gabriela Caldeira do Espírito Santo \\ ORCID: https://orcid.org/0000-0002-0084-2318 \\ Universidade Federal de Sergipe, Brasil \\ E-mail: gbcaldeira1233@gmail.com \\ Walderi Monteiro da Silva Júnior \\ ORCID: https://orcid.org/0000-0002-6815-4386 \\ Universidade Federal de Sergipe, Brasil \\ E-mail: walderim@yahoo.com.br \\ Jader Pereira de Farias Neto \\ ORCID: https://orcid.org/0000-0003-2781-6870 \\ Universidade Federal de Sergipe, Brasil \\ E-mail: jadernetofisio@hotmail.com
}

\section{Resumo}

Objetivo: Avaliar o efeito de um programa de educação em dor associado à terapia com exercícios em pacientes que tenham dor lombar crônica inespecífica com e sem sensibilização central. Metodologia: Trata-se de um ensaio clínico controlado aleatorizado, em que os pacientes foram alocados em dois grupos: pacientes com dor lombar crônica inespecífica sem sensibilização central $(\mathrm{G} 1)$ ou pacientes com dor lombar crônica inespecífica com sensibilização (G2). Após avaliação prévia da intensidade da dor, percepção de melhora, incapacidade, cinesiofobia, agilidade e gastos em saúde, os pacientes receberam o tratamento durante 5 semanas, totalizando 10 sessões. Resultados: Os resultados não apontaram diferenças entre os grupos, mas na comparação intragrupo percebeu-se melhora da mobilidade $(\mathrm{p}=0,005)$ e dor $(\mathrm{p}=0,005)$ e redução da incapacidade $(\mathrm{p}=0,036)$ e cinesiofobia $(\mathrm{p}=0,012)$ para o G1. Para o G2 houve melhora da mobilidade $(\mathrm{p}=0,012)$, da dor $(\mathrm{p}=0,012)$ e redução da incapacidade $(\mathrm{p}=0,018)$. O nível de atividade física foi diferente apenas para o grupo com sensibilização central $(p=0,034)$. Quanto aos gastos em saúde, percebe-se que não houve modificações ao longo do tempo, para internações e gastos com consultas médicas, visto o curto período avaliado. Conclusão: Conclui-se que, um programa de educação em dor combinado com exercícios é benéfico para melhora da funcionalidade física, dor, redução da incapacidade e cinesiofobia para o G1, enquanto, para o G2 houve melhora do nível de atividade física, mobilidade, dor e redução da incapacidade. Por fim, esperamos que esse estudo contribua para a implantação de programas e protocolos de atendimento mais eficazes.

Palavras-chave: Dor lombar; Sensibilização do sistema nervoso central; Terapia por exercício; Educação da população; Manejo da dor.

\footnotetext{
Abstract

Objective: To evaluate the effect of a pain education program associated with exercise therapy in patients who have chronic nonspecific low back pain with and without central sensitization. Methodology: This is a randomized controlled clinical trial, in which patients were divided into two groups: patients with chronic nonspecific low back pain without central sensitization (G1) or patients with nonspecific chronic low back pain with sensitization (G2).
} 
After previous assessment of pain intensity, perception of improvement, disability, kinesiophobia, agility and health expenses, patients received treatment for 5 weeks, totaling 10 sessions. Results: The results did not show differences between the groups, but the intragroup comparison showed an improvement in mobility $(p=0.005)$ and pain $(p=0.005)$ and a reduction in disability $(\mathrm{p}=0.036)$ and kinesiophobia $(\mathrm{p}=0.012)$ for the $\mathrm{G} 1$. For $\mathrm{G} 2$ there was improvement in mobility $(\mathrm{p}=0.012)$, pain $(\mathrm{p}=0.012)$ and reduction in disability $(\mathrm{p}=0.018)$. The level of physical activity was different only for the group with central sensitization $(p=0.034)$. As for health expenses, it is clear that there were no changes over time, for hospitalizations and expenses with medical appointments, given the short period evaluated. Conclusion: It is concluded that a pain education program combined with exercise is beneficial to improve physical functionality, pain, reduced disability and kinesiophobia for G1, while for G2 there was an improvement in the level of physical activity, mobility, pain and disability reduction. Finally, we hope that this study will contribute to the implementation of more effective care programs and protocols.

Keywords: Low back pain; Sensitization of the central nervous system; Exercise therapy; Education of the population; Pain management.

\section{Resumen}

Objetivo: Evaluar el efecto de un programa de educación sobre el dolor asociado con la terapia de ejercicios en pacientes que tienen dolor lumbar crónico inespecífico con y sin sensibilización central. Metodología: Se trata de un ensayo clínico controlado aleatorizado, en el que los pacientes se dividieron en dos grupos: pacientes con lumbalgia crónica inespecífica sin sensibilización central $(\mathrm{G} 1)$ ou pacientes con lumbalgia crónica inespecífica con sensibilización (G2). Después de una evaluación previa de la intensidad del dolor, percepción de mejoría, discapacidad, kinesiofobia, agilidad y gastos de salud, los pacientes recibieron tratamiento durante 5 semanas, totalizando 10 sesiones. Resultados: Los resultados no mostraron diferencias entre los grupos, pero la comparación intragrupo mostró una mejora en la movilidad $(\mathrm{p}=0,005)$ y el dolor $(\mathrm{p}=0,005)$ y una reducción en la discapacidad $(\mathrm{p}$ $=0,036)$ y kinesiofobia $(\mathrm{p}=0,012)$. para el G1. Para G2 hubo mejoría en la movilidad $(\mathrm{p}=0.012)$, dolor $(\mathrm{p}=0.012)$ y reducción de la discapacidad $(\mathrm{p}=0.018)$. El nivel de actividad física fue diferente solo para el grupo con sensibilización central $(\mathrm{p}=0.034)$. En cuanto a los gastos en salud, es claro que no hubo cambios en el tiempo, para las hospitalizaciones y los gastos con citas médicas, dado el corto período evaluado. Conclusión: Se concluye que un programa de educación del dolor combinado con ejercicio es beneficioso para mejorar la funcionalidad física, el dolor, la disminución de la discapacidad y la kinesiofobia para G1, mientras que para G2 hubo una mejora en el nivel de actividad física, movilidad, dolor y reducción de la discapacidad. Finalmente, esperamos que este estudio contribuya a la implementación de programas y protocolos de atención más efectivos.

Palabras clave: Lumbalgia; Sensibilización del sistema nervioso central; Terapia de ejercicio; Educación de la población; El manejo del dolor.

\section{Introdução}

A dor lombar (DL) é a doença musculoesquelética mais comum globalmente, além de ser a principal causa de inatividade, com prevalência no tempo de vida variando entre 80\% e 85\% (Hoy et al., 2014; Wu et al., 2020). Ademais, a limitação e absenteísmo resultantes dessa problemática, envolvem grande demanda médica e alto custo econômico, fazendo com que este seja um dos principais problemas de saúde pública em todo mundo (Wu et al., 2020).

Parte da população com dor lombar crônica também pode apresentar vários sintomas desproporcionais, como hiperalgesia, além de sintomas somáticos e psicossociais. Isso ocorre em virtude indução de estímulos nocivos periféricos crônicos, causando um desequilíbrio nos neurotransmissores centrais excitatórios e inibitórios e alterando as regulações nesse sistema, levando à hiperexcitabilidade e consequente sensibilização central (Aoyagi et al., 2019). Para alguns indivíduos, crenças e informações negativas com diagnósticos imprecisos podem ser somados à catastrofização da dor e terem tal efeito potencializador para dor (Manning et al., 2020; Ross et al., 2017).

Sabe-se que indivíduos com dor lombar crônica gastam 1,7 vez mais com medicamentos, 2,4 vezes mais com consultas, 2,1 vezes mais com hospitalizações e 4,2 vezes mais com consultas de emergência, quando comparados a indivíduos controle (Gore et al., 2012; Miyamoto et al., 2019). Embora o tema seja amplamente estudado, o gerenciamento de DL inespecífica torna-se complexa devido as contribuições e consequências multidimensionais, incluindo fatores biológicos, psicológicos e sociais (Tagliaferri et al., 2020).

De acordo com Kolu, et al. (2017) uma maneira de reduzir os custos em saúde é aumentando o nível de atividade física dos pacientes com dor lombar crônica. Este autor sugere que, quando os pacientes atingem níveis de aptidão muscular e 
cardiorrespiratória considerados satisfatórios, a média de custos totais com serviços de saúde é cerca de $80 \%$ menor. Com base nos dados que demonstram que o descondicionamento geral, isolamento social e sofrimento psicológico estão relacionados com a lombalgia, Steffens, et al. (2016), destacam que a combinação dos exercícios globais e da educação do paciente é capaz de prevenir a sintomatologia incapacitante. Para mais, parece dessensibilizar o sistema nervoso à medida que muda as crenças e comportamentos, reduzindo distorções cognitivas (Pardo et al.,2018).

Além disso, abordagens como a educação em neurociência da dor e treinamento físico, têm demostrado bons resultados na redução da intensidade da dor. A educação neurofisiológica visa mudar as atitudes e crenças relacionadas à dor, explicando os indivíduos acerca dos fatores cognitivos, sociais e contextuais que modulam a dor, através de uma abordagem biopsicossocial (Traeger et al., 2014). Ademais, as diretrizes recomendam a utilização da terapia ativa, por meio de exercícios, mesmo existindo divergência na literatura sobre intensidade, duração e indicação biomecânica dos exercícios mais apropriados para esses indivíduos (Airaksinen et al., 2006; Foster et al., 2018).

Baseado no exposto, a educação do paciente tornou-se tratamento de primeira linha, pois é simples e de baixo custo, especialmente em adição a terapia com exercícios, já que nenhuma terapia isolada é eficiente (Silva \& Martins, 2014). No cenário do Sistema Único de Saúde (SUS), essas abordagens podem, além de melhorar a dor e função, promover a diminuição das filas de espera, uma vez que têm a capacidade de alterar a atividade das áreas cerebrais relacionadas a modulação da dor e as crenças negativas sobre o movimento e por consequência aumentar o nível de atividade física (Moseley, 2005; Wood \& Hendrick, 2019).

Dessa forma, o objetivo do estudo foi avaliar o efeito de um programa de educação em dor associado à terapia com exercícios na agilidade e mobilidade em pacientes que tenham dor lombar crônica inespecífica com e sem sensibilização central, na intensidade da dor, incapacidade, cinesiofobia, catastrofização, percepção de melhora, nível de atividade física e gastos em saúde.

\section{Metodologia}

Trata-se de ensaio clínico piloto controlado não aleatorizado (Estrela, 2018), aprovado pelo Comitê de Ética em Pesquisa com Seres Humanos da Universidade Federal de Sergipe (Protocolo: 2.412.335). Neste estudo, os pacientes foram alocados em um dos dois grupos de acordo com a sua categorização no Inventário de Sensibilização Central (Caumo et al., 2017). Ainda, o avaliador foi do tipo cego e dada a natureza das intervenções, o paciente e o terapeuta que acompanhou o tratamento, não foram cegos. Além disso, todos os procedimentos foram realizados conforme Resolução no. 466/12 do Conselho Nacional de Saúde. Participaram deste estudo, indivíduos com diagnóstico de dor lombar com 3 meses de duração, ou mais, idade entre 18 e 75 anos e intensidade de dor $\geq 3$ na escala numérica da dor. Foram critérios de exclusão: qualquer contraindicação ao exercício físico, declínio cognitivo, patologia espinal grave, cirurgia espinhal anterior, comprometimento da raiz do nervo, doenças cardiorrespiratórias ou gravidez.

Inicialmente foram avaliados dados antropométricos, como peso e altura, e intensidade da dor, pela Escala Numérica da Dor (Chiarotto, Terwee \& Ostelo, 2017). Em seguida foram aplicados os questionários de Roland Morris Disability Questionnaire (RMDQ), para incapacidade (Chiaroto, et al., 2017), Fear Avoidance Beliefs Questionnaire, para cinesiofobia (Abreu et al., 2008; Costa et al., 2008), Escala de Catastrofização da dor, para catastrofização da dor (Sehn, Caumo \& Souza, 2012), e Inventário de Sensibilização Central, para risco de sensibilização central (Caumo et al., 2017).

Para avaliar a mobilidade da coluna, foi utilizado o teste dedos ao solo. Foi adotado o modelo de mensuração proposto por Magnusson et al., (1997), onde aqueles que conseguirem atingir uma distância inferior a dez centímetros em relação ao chão ou toque no chão serão classificados como flexibilidade normal, e os que atingirem valores maiores que dez centímetros serão classificados como flexibilidade reduzida. Também, utilizou-se a Escala da Percepção do Efeito Global (EPEG - GPE), 
para avaliação da percepção do paciente quanto a terapia empregada (Costa et al., 2008). Os gastos em saúde foram avaliados por meio de perguntas, que seguiram o modelo proposto por Zanuto et al. (2017), coletados na primeira avaliação. Foi perguntado ao paciente: 1. No último ano, o sr./sra. precisou ser internado? 2. No último ano, o sr./sra. fez alguma cirurgia? 3. Por quanto tempo ficou internado ou impossibilitado de exercer suas funções cotidianas? 4. Precisou faltar ao trabalho por conta de internação? 5. O seu tratamento foi pago pelo SUS, particular ou por convênio? 6. Qual foi o local onde realizou o tratamento? 7. No último ano, o sr./sra. foi na emergência por causa da dor?

Os pacientes foram alocados em dois grupos: pacientes com dor lombar crônica sem sensibilização central (G1) e pacientes com dor lombar e com sensibilização central (G2). Foram realizadas dez sessões de uma hora durante cinco semanas, duas vezes por semana. Cada sessão será dividida em até 20 minutos de aulas teóricas e 40 minutos de exercícios funcionais de flexibilidade, mobilidade e força, prescritos e progredidos a cada semana de tratamento (Quadro 1). As aulas teóricas foram baseadas no livro "Explicando a dor" do autor Rodrigo Rizzo. Ao final de cada sessão os indivíduos foram avaliados quanto a dor, percepção de melhora e realizaram o teste dedos ao chão para monitoramento da mobilidade lombar. No decorrer das sessões os indivíduos foram analisados para que na última aula fossem encaminhados para projetos sociais adequados ao seu perfil.

Quadro 1 - Protocolo de tratamento.

\begin{tabular}{|c|c|c|c|c|c|}
\hline Exercício & Semana 1 & Semana 2 & Semana 3 & Semana 4 & Semana 5 \\
\hline 4 apoios & $\begin{array}{c}\text { Retirada de } 1 \\
\text { mmss }\end{array}$ & $\begin{array}{c}\text { Retirada } 1 \\
\text { mmii }\end{array}$ & $\begin{array}{c}\text { Retirada de } 1 \\
\text { mmss e } 1 \mathrm{mmii}\end{array}$ & $\begin{array}{c}\text { Retirada de } 1 \\
\text { mmss e } 1 \mathrm{mmii} \\
\text { com } \\
\text { manutenção } \\
\text { durante } 10 \\
\text { segundos }\end{array}$ & $\begin{array}{c}\text { Retirada de } 1 \\
\text { mmss e } 1 \text { mmii } \\
\text { com manutenção } \\
\text { de } 30 \text { segundos }\end{array}$ \\
\hline Ponte & Ponte simples & $\begin{array}{c}\text { Ponte simples } \\
\text { com resistência } \\
\text { elástica } \\
\end{array}$ & $\begin{array}{l}\text { Ponte simples } \\
\text { com abdução }\end{array}$ & $\begin{array}{l}\text { Ponte unipodal } \\
\text { com flexão de } \\
\text { joelho }\end{array}$ & $\begin{array}{l}\text { Ponte unipodal } \\
\text { com extensão de } \\
\text { joelho }\end{array}$ \\
\hline Agachamento & $\begin{array}{l}\text { Sentar e } \\
\text { levantar }\end{array}$ & $\begin{array}{l}\text { Agachamento } \\
\text { livre }\end{array}$ & $\begin{array}{c}\text { Agachamento } \\
\text { com arremesso } \\
\text { de bola }\end{array}$ & $\begin{array}{l}\text { Agachamento } \\
\text { com extensão } \\
\text { unipodal da } \\
\text { perna }\end{array}$ & $\begin{array}{c}\text { Agachamento com } \\
\text { deslocamento } \\
\text { lateral }\end{array}$ \\
\hline Abdominais & $\begin{array}{l}\text { Prancha frontal } \\
\text { com apoio das } \\
\text { mãos e joelhos }\end{array}$ & $\begin{array}{c}\text { Prancha lateral } \\
\text { com apoio dos } \\
\text { joelhos }\end{array}$ & $\begin{array}{l}\text { Prancha frontal } \\
\text { com apoio das } \\
\text { mãos e pés }\end{array}$ & $\begin{array}{c}\text { Prancha lateral } \\
\text { com apoio dos } \\
\text { pés }\end{array}$ & $\begin{array}{l}\text { Prancha frontal } \\
\text { comum }\end{array}$ \\
\hline $\begin{array}{l}\text { Mobilizações } \\
\text { ativas com } \\
\text { movimento }\end{array}$ & $\begin{array}{c}\text { Báscula pélvica } \\
\text { em DD }\end{array}$ & $\begin{array}{l}\text { DD com } \\
\text { rotação do } \\
\text { quadril } \\
\text { lateralmente }\end{array}$ & Gato e camelo & $\begin{array}{c}\text { Torácica em } \\
\text { quatro apoios }\end{array}$ & $\begin{array}{l}\text { Rotação de torácica } \\
\text { em quadrupedia }\end{array}$ \\
\hline Alongamentos & $\begin{array}{c}\text { Rotadores } \\
\text { externos } \\
\text { Isquiotibiais } \\
\text { Iliopsoas } \\
\end{array}$ & $\begin{array}{l}\text { Rotadores } \\
\text { externos } \\
\text { Isquiotibiais } \\
\text { Iliopsoas } \\
\end{array}$ & $\begin{array}{l}\text { Rotadores } \\
\text { externos } \\
\text { Isquiotibiais } \\
\text { Iliopsoas } \\
\end{array}$ & $\begin{array}{l}\text { Rotadores } \\
\text { externos } \\
\text { Isquiotibiais } \\
\text { Iliopsoas } \\
\end{array}$ & $\begin{array}{l}\text { Rotadores externos } \\
\text { Isquiotibiais } \\
\text { Iliopsoas }\end{array}$ \\
\hline \multirow[t]{4}{*}{ Aulas } & Aula 1 & Aula 3 & Aula 5 & Aula 7 & Aula 9 \\
\hline & A dor é normal & $\begin{array}{c}\text { Alteração do } \\
\text { sistema de } \\
\text { alarme } \\
\end{array}$ & $\begin{array}{l}\text { Hormônios e } \\
\text { sistema }\end{array}$ & $\begin{array}{l}\text { Seu diagnóstico } \\
\text { não é o fím }\end{array}$ & $\begin{array}{c}\text { Adaptações e } \\
\text { mudança no estilo } \\
\text { de vida }\end{array}$ \\
\hline & Aula 2 & Aula 4 & Aula 6 & Aula 8 & Aula 10 \\
\hline & $\begin{array}{l}\text { O sistema de } \\
\text { alarme }\end{array}$ & $\begin{array}{l}\text { Influência das } \\
\text { emoções e } \\
\text { pensamentos }\end{array}$ & $\begin{array}{l}\text { Manejo da dor } \\
\text { e Estilo de vida }\end{array}$ & $\begin{array}{c}\text { Mitos } \\
\text { relacionados a } \\
\text { dor }\end{array}$ & $\begin{array}{l}\text { Evite as crises e } \\
\text { tire suas dúvidas }\end{array}$ \\
\hline
\end{tabular}

Fonte: Autores. 
Os dados foram catalogados na forma de arquivos de planilha de cálculo do Microsoft Excel® e para o tratamento estatístico foi utilizado o programa Statistical Package for the Social Sciences (SPSS versão 25). A condição de normalidade foi avaliada pelo teste de Shapiro-Wilk. Foi utilizado o teste t de Student para comparação dos dados paramétricos e o teste Mann Whitney para dados não paramétricos. Para as variáveis categóricas a comparação foi realizada com o teste Qui quadrado. As comparações foram realizadas intra e intergrupos. Valores de $p<0,05$ indicaram significância estatística. Para a análise do intergrupo foi realizada a comparação da diferença entre pós e pré intervenção. Já a análise qualitativa de gastos foi realizada por meio de condensação de significantes.

\section{Resultados}

Foram convidados 52 pacientes, todavia apenas 19 finalizaram o estudo, destes 10 eram do G1 e 9 do G2. Os dados estão representados no fluxograma (Figura 1).

Figura 1 - Fluxograma da seleção dos indivíduos.

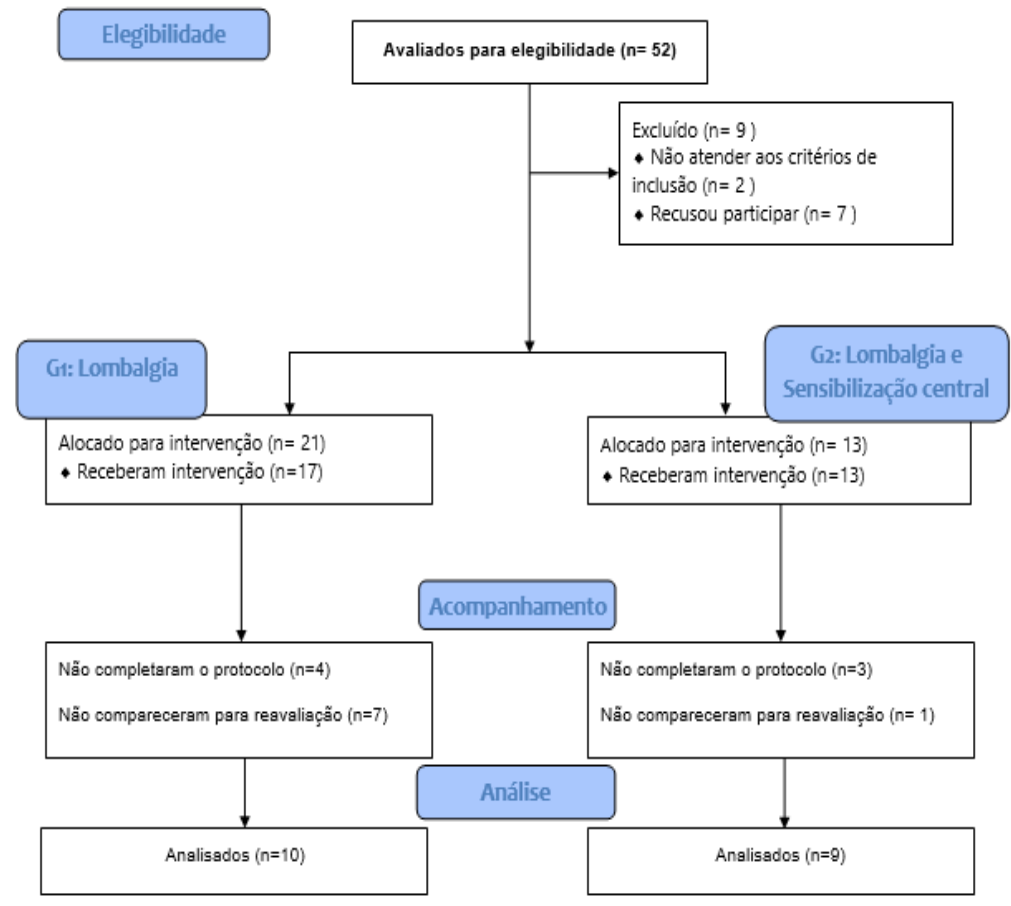

Fonte: Autores.

As características desses pacientes encontram-se descritas na Tabela 1. 
Tabela 1 - Caracterização da amostra.

\begin{tabular}{lccc}
\hline & $\begin{array}{c}\text { G1: } \\
\text { Lombalgia }\end{array}$ & $\begin{array}{c}\text { G2: Lombalgia e } \\
\text { Sensibilização }\end{array}$ & p \\
\hline Idade & $58,36 \pm 15,57$ & $53,38 \pm 9,59$ & $0,60^{\mathrm{a}}$ \\
Sexo & & & \\
$\quad$ Feminino (n (\%)) & $8(80 \%)$ & $7(77,7 \%)$ & $0,12^{\mathrm{b}}$ \\
Altura (m) & $1,57 \pm 0,07$ & $1,58 \pm 0,07$ & $0,96^{\mathrm{a}}$ \\
Peso (kg) & $71,9 \pm 15,56$ & $80,1 \pm 12,08$ & $0,31^{\mathrm{a}}$ \\
IMC (kg/m $\left.{ }^{2}\right)$ & $28,82 \pm 5,18$ & $31,94 \pm 4,32$ & $0,17^{\mathrm{a}}$ \\
Nível de atividade Física (IPAQ) & & & \\
$\quad$ Ativo (n (\%)) & $2(18,2 \%)$ & $7(87,5 \%)$ & $0,005^{\mathrm{b} *}$ \\
$\quad$ Muito ativo (n (\%)) & $6(54,5 \%)$ & $1(12,5 \%)$ & \\
$\quad$ Sedentário (n (\%)) & $3(27,3 \%)$ & 0 & $0,54^{\mathrm{a}}$ \\
\hline Intensidade da dor (EVA) & $7,18 \pm 2$ & $7 \pm 1$ & \\
\hline
\end{tabular}

Legenda: m: metro; kg: quilograma; IMC: Índice de massa corpórea; IPAQ: Questionário Internacional de Atividade Física; EVA: escala visual analógica da dor na primeira avaliação; a: comparação realizada com teste U de Mann-Whitney; b: comparação realizada com o teste Qui quadrado; *: $p<0,05$. Fonte: Autores.

A comparação entre os grupos não foi significativa para nenhuma das variáveis (Tabela 2).

Tabela 2: Parâmetros do estudo para G1 e G2.

\begin{tabular}{|c|c|c|c|c|c|c|c|}
\hline \multirow[t]{2}{*}{ Variável } & \multicolumn{2}{|c|}{ G1: Lombalgia } & \multicolumn{2}{|c|}{$\begin{array}{l}\text { G2: Lombalgia e } \\
\text { Sensibilização }\end{array}$} & \multicolumn{2}{|c|}{$\begin{array}{c}\text { Comparação } \\
\text { intragrupo } \\
\text { (antes - depois) }\end{array}$} & \multirow{2}{*}{ 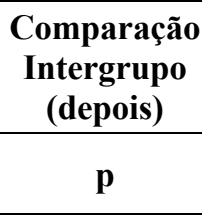 } \\
\hline & Pré & Pós & Pré & Pós & $\begin{array}{c}\text { G1 } \\
\mathbf{p}\end{array}$ & $\begin{array}{c}\text { G2 } \\
\text { p }\end{array}$ & \\
\hline TC6 (m) & $332,54 \pm 129,7$ & $353,4 \pm 107,5$ & $325,6 \pm 69,4$ & $359,5 \pm 41,6$ & 0,131 & 0,161 & 0,483 \\
\hline TUG (s) & $12,22 \pm 8,8$ & $10 \pm 1,10$ & $10,35 \pm 1,8$ & $9,58 \pm 2,28$ & 0,476 & 0,398 & 0,649 \\
\hline Mobilidade & $18,36 \pm 13,25$ & $13,83 \pm 11,41$ & $27,56 \pm 17,73$ & $18,5 \pm 13,37$ & $0,005^{*}$ & $0,012^{*}$ & 0,322 \\
\hline Dor (EVA) & $7,18 \pm 2$ & $2,73 \pm 3$ & $7 \pm 1$ & $3,38 \pm 2,44$ & $0,005^{*}$ & $0,012 *$ & 0,402 \\
\hline RMDQ & $14 \pm 6,23$ & $10,91 \pm 6,8$ & $16,38 \pm 3,02$ & $12,5 \pm 4,8$ & $0,036^{*}$ & $0,018^{*}$ & 0,481 \\
\hline $\begin{array}{c}\text { FABQ } \\
\text { ATIVIDADE }\end{array}$ & $12,9 \pm 9,91$ & $3,82 \pm 2$ & $18,8 \pm 10$ & $8,13 \pm 10$ & $0,012 *$ & 0,075 & 0,293 \\
\hline $\begin{array}{c}\text { FABQ } \\
\text { TRABALHO }\end{array}$ & $46,27 \pm 16,3$ & $34,6 \pm 18,4$ & $37,3 \pm 23,4$ & $41,3 \pm 22,1$ & $0,029 *$ & 0,310 & 0,385 \\
\hline ECD & $27,6 \pm 16,4$ & $18 \pm 10$ & $32,8 \pm 9,8$ & $26,7 \pm 16,2$ & 0,141 & 0,159 & 0,283 \\
\hline $\begin{array}{l}\text { Percepção de } \\
\text { melhora }\end{array}$ & $4,36 \pm 1,56$ & $3,73 \pm 1,47$ & $3,75 \pm 1,8$ & $4,75 \pm 0,46$ & 0,068 & 0,059 & 0,058 \\
\hline
\end{tabular}

Legenda: m - metros; s - segundos; TC6 - Teste de Caminhada de 6 minutos; TUG - Teste Time Up and Go; EVA - Escala Visual Analógica; RMDQ - Questionário Rolland Morris Disability;; FABQ - ATIVIDADE - Questionário Fear Avoidance Beliefs subescala Atividade Física; FABQ - TRABALHO - Questionário Fear Avoidance Beliefs subescala Trabalho; ECD

- Escala de Catastrofização da Dor; * - $\mathrm{p}<0,05$. Fonte: Autores.

Para a comparação intragrupo também não foram encontradas diferenças para os testes de agilidade nem para o grupo lombalgia (TC6 - Z = - 1,511, p =0,131; TUG - Z = - 0,713, p =0,476) nem para o grupo com lombalgia e sensibilização central (TC6 - Z = - 1,40, p =0,161; TUG - Z = - 0,845, p=0,398). Entretanto, para os pacientes com lombalgia é possível perceber melhora da mobilidade de coluna $(Z=-2,803, p=0,005)$, redução da dor $(Z=-2,807, p=$ 0,005), da incapacidade $(Z=-2,098, p=0,036)$, e da cinesiofobia (atividade física $-Z=-2,521, p=0,012 ;$ trabalho $-Z=$ 
- 2,180, $\mathrm{p}=0,029)$. Apenas a escala de catastrofização da dor $(Z=-1,472, p=0,141)$ e percepção de melhora $(Z=-$ $1,823, \mathrm{p}=0,068)$ não apresentaram diferenças significativas.

Para o grupo com lombalgia e sensibilização central, a melhora da mobilidade $(Z=-2,521, p=0,012)$ e da dor $(Z=-2,527, p=0,012)$ apresentaram diferenças significativas. De forma semelhante, os níveis de incapacidade reduziram significativamente $(Z=-2,371, p=0,018)$. Entretanto, a redução de cinesiofobia tanto nas escalas de atividade física $(Z=-1,778, p=0,075)$, quanto para trabalho $(Z=-1,014, p=0,310)$ não foi significativa. Similarmente a escala de catastrofização $(Z=-1,407, p=0,159)$, e a percepção de melhora $(Z=-1,890, p=0,059)$ não foram significativos.

Em relação a avaliação do nível de atividade física na comparação intragrupo, notou-se que indivíduos com sensibilização central se apresentavam majoritariamente como ativos na primeira avaliação, o que era estatisticamente significante. Entretanto, em ambos os grupos, a distribuição desses pacientes foi diluída entre as classificações do IPAQ não apresentando diferenças ao final do tratamento (Tabela 3).

Tabela 3: Avaliação do nível de atividade física (IPAQ).

\begin{tabular}{cccccc}
\hline & & Sedentário & Ativo & Muito ativo & p \\
\hline Lombalgia & Pré & $3(27,3 \%)$ & $6(54,5 \%)$ & $2(18,2 \%)$ & 0,307 \\
& Pós & $1(9,09 \%)$ & $7(63,6 \%)$ & $3(27,3 \%)$ & 0,078 \\
\multirow{2}{*}{ Lombalgia e sensibilização central } & Pré & 0 & $7(87,5 \%)$ & $1(12,5 \%)$ & $0,034^{*}$ \\
& Pós & $3(37,5 \%)$ & $4(50 \%)$ & $1(12,5 \%)$ & 0,417 \\
\hline
\end{tabular}

IPAQ - Questionário Internacional de Atividade Física; * - p<0,05. Fonte: Autores.

Quanto aos gastos em saúde é possível perceber que os pacientes apresentaram respostas que não diferiam ao longo do tempo. Cerca de $85 \%$ dos pacientes não haviam sido internados nos últimos 12 meses, e $89 \%$ não fizeram cirurgia. Aproximadamente $79 \%$ dos pacientes realizavam algum tipo de tratamento antes da intervenção com educação em dor e $73 \%$ precisaram de atendimento de emergência. Os resultados de antes e depois podem ser vistos na Tabela 4.

Tabela 4: Comparação entre os momentos antes e depois da intervenção quanto aos gastos em saúde.

\begin{tabular}{llll}
\hline & & Antes & Depois \\
\hline $\begin{array}{l}\text { Você foi internado no } \\
\text { último ano? }\end{array}$ & Sim & $3(15,7 \%)$ & $3(15,7 \%)$ \\
& Não & $16(84,2 \%)$ & $16(84,2 \%)$ \\
$\begin{array}{l}\text { Você fez alguma cirurgia no } \\
\text { último ano? }\end{array}$ & Sim & $2(10,5 \%)$ & $2(10,5 \%)$ \\
& Não & $17(89,5 \%)$ & $17(89,5 \%)$ \\
$\begin{array}{l}\text { Fez algum tratamento para } \\
\text { coluna no último ano? }\end{array}$ & Sim & $15(79 \%)$ & $19(100 \%)$ \\
$\begin{array}{l}\text { Precisou de atendimento de } \\
\text { emergência no último ano? }\end{array}$ & Simão & $4(21 \%)$ & 0 \\
\hline
\end{tabular}

Fonte: Autores.

Quando questionados sobre quais recursos da saúde foram utilizados, os participantes responderam principalmente o uso de medicamento sendo comprado pelos próprios usuários (tabela 5). Entretanto, foram considerados também acompanhamento médico e o tratamento fisioterapêutico. 
Tabela 5: Relatos dos pacientes quando perguntados sobre os tratamentos realizados para dor nas costas no último ano.

\begin{tabular}{lcc}
\hline Tratamento & Modalidade & $\begin{array}{c}\text { Quantidade de pacientes } \\
(\mathbf{n}=\mathbf{1 9})\end{array}$ \\
\hline Médico & Particular & $1(5,26 \%)$ \\
& Sistema único de Saúde & $5(26,3 \%)$ \\
Fisioterapia & Convênio & $1(5,26 \%)$ \\
& Particular & $1(5,26 \%)$ \\
Remédio & Sistema Único de Saúde & $5(26,3 \%)$ \\
Não Realizaram Tratamento & & $6(31,57 \%)$ \\
\hline
\end{tabular}

Fonte: Autores.

Ademais, dos 19 pacientes atendidos, apenas seis procuraram auxílio médico, sete indivíduos fisioterapia ou outros tratamentos ligados a essa modalidade e cerca de seis utilizaram medicamentos. A única alteração que se percebe neste ponto da avaliação qualitativa se dá sobre o tratamento para coluna no período de um ano. Isso se dá pelo curto período do tratamento, visto que a reavaliação foi feita apenas após as cinco semanas, não há grande influência sobre o que se passou nos últimos 12 meses. Sugerimos, nesse ponto, o acompanhamento desses pacientes por um intervalo de tempo maior, para que seja avaliado o impacto a longo prazo.

\section{Discussão}

A dor lombar é um importante problema de saúde em ascensão em todo o mundo. Dada a sua heterogeneidade, dificuldade de diagnóstico e consequente resultados insatisfatórios, o objetivo primário desse estudo foi avaliar o efeito de um programa de educação em dor na agilidade e mobilidade em pacientes com e sem sensibilização central que tenham dor lombar. Apesar dos resultados não terem sido significativos quando se compara as duas condições de saúde estabelecidas, há uma melhora importante em cada uma delas, principalmente quando se leva em consideração os indicadores de intensidade da dor, mobilidade e incapacidade.

Sabe-se que a catastrofização da dor pode estar associada à intensidade, incapacidade, e evitação relacionados ao movimento, gerando desuso e reafirmando diversos sintomas prejudiciais (Marques, 2020). Nossos resultados tanto intragrupo, como intergrupo não demonstraram diferenças significativas na escala de catastrofização da dor e percepção de melhora. De acordo com o estudo de Meints et al. (2019), o nível de catastrofização está associada ao surgimento da dor e a sensibilidade relacionada a ela em indivíduos crônicos, assim, induz maior hiperalgesia do tecido profundo, aproximando a relação entre catastrofização e dor entre esses pacientes. Nesse sentido, acreditamos que mesmo ao dividirmos a amostra entre pacientes com sensibilização central e àqueles sem, foi possível notar semelhanças à sua resposta à terapia, devido aos fatores biopsicossociais envolvidos.

Ao ressaltarmos o modelo biopsicossocial, notamos que fatores cognitivos, emocionais, psicológicos, comportamentais, físicos e sociais interagem para perpetuação da dor (Igwesi-Chidobe et al., 2017). Além disso, a percepção de melhora é uma variável subjetiva, que requer do paciente uma boa memória antes e após o tratamento. Em casos de dor crônica, essa percepção pode ser alterada, e com a adição do viés de recordação do paciente, o julgamento retrospectivo da doença pode ser incorreto, relacionando-se mais com o status atual do que com uma melhora temporal (Maughan \& Lewis, 2010). Em associação, a persistência da cinesiofobia e alteração de função associada a dor, também pode ocorrer, mesmo após tratamento fisioterapêutico, uma vez que está diretamente relacionada com as crenças do paciente sobre sua disfunção (Kim \& Lee, 2020). 
Vale destacar que diversos estudos apresentam diferenças quanto ao processamento da dor nesse tipo de paciente, todavia, esses estudos comparam indivíduos saudáveis com àqueles sensibilizados (Meints et al., 2019; Jensen, Nielsen E Stengaard-Pedersen, 2010; O’Neill et al., 2007), diferentemente da nossa amostra, que foi composta por indivíduos com sintomas crônicos. De maneira similar aos nossos resultados, durante estudo transversal, Almeida et al. (2021), demonstram que pacientes sensibilizados centralmente, apresentam menor desempenho físico, maiores taxas de incapacidade, cinesiofobia, catastrofização da dor e menor qualidade de vida em comparação com aqueles com menor risco de sensibilização.

Devido as diferenças culturais, podem existir alterações nas percepções da doença, de modo que, pacientes com menor grau de instruções sobre seu quadro clínico, com vários diagnósticos específicos e diversos tratamentos com promessa de cura, podem desenvolver maior preocupação com a doença quando a resolução dos sintomas não foi alcançada. Ademais, percepções acerca da doença são um dos principais determinantes para a deficiência autorrelatada em casos de dor lombar (Igwesi-Chidobe et al., 2017).

Em contraste, pacientes que experimentam a autogestão com base na educação sobre a natureza da dor crônica apresentam melhores resultados num período menor que 12 semanas (Wood, Lianne \& Hendrick, 2019). Ainda, pacientes que receberam vários tipos tratamentos em que nenhum deles resolveu seu problema, costumam ter altos níveis de sofrimento psicológico (Walker, Sofaer \& Holloway, 2006). Esse é um fato notado, visto que, apesar do nosso estudo ser de curto prazo, 6 semanas, acreditamos que dada a complexidade dos indivíduos e seu tempo de espera na fila, a terapia combinada não foi suficiente para alterar sua percepção, pois grande parte já havia experimentado diversos tipos de conduta, sejam medicamentosas ou físicas. Embora o estudo de Foster et al. (2010), tenha notado esse comportamento mais evidente à nível terciário em países mais ricos, em países em desenvolvimento, como o Brasil, cerca de $94 \%$ dos indivíduos que apresentam dor na região lombar apresentam déficits funcionais, aumentando a chance de procura por tratamento (Barbosa et al., 2019).

No grupo de indivíduos apenas com lombalgia, notou-se melhora da mobilidade de coluna, redução da dor, da incapacidade, e da cinesiofobia. Corroborando os nossos achados, Pardo et al. (2018), notaram que em uma amostra com dor lombar crônica, a terapia combinada de educação com exercícios, produziu melhoras significativamente maiores na intensidade da dor, deficiência, fatores psicossociais e desempenho físico quando em comparação com a realização apenas de exercícios. Consoante a revisão de Wong et al. (2016) com base em diretrizes de alta qualidade metodológica, os pacientes com dor lombar devem receber educação neurofisiológica da dor e serem incentivados a permanecerem ativos, bem como retornar à atividade conforme tolerado. Além disso, fica comprovado que a maioria das modalidades passivas, como laser e ultrassom, não são recomendadas para o tratamento da dor lombar crônica (Wong et al., 2016).

Apesar da terapia combinada ser considerada benéfica, é provável que no nosso estudo, melhores resultados não tenham sido alcançados em decorrência do curto período de tratamento. No estudo de Semedo (2017), a mediana do tempo de recuperação para a intensidade da dor e da incapacidade funcional, ocorreu entre 6.3 e 6.5 semanas, já no trabalho de Verkerk et al. (2013), 24.1\%, cerca de 409 indivíduos, reportaram recuperação total apenas dois meses após a avaliação inicial, coincidente com o término da intervenção. De forma semelhante, os níveis de incapacidade reduziram significativamente, favorecendo a realização das atividades cotidianas. Os sintomas típicos da dor lombar, ocasionam inibição do movimento a longo prazo e inatividade física que pode levar a alterações fisiológicas e neurológicas, como fraqueza da musculatura paravertebral, e alteração da resposta de relaxamento da musculatura da coluna (Gasibat et al., 2019).

De certo, essas restrições ocorrem principalmente de maneira intencional, pois os indivíduos restringem atividades por medo de causar danos ou lesões, assim, objetivo do exercício é diminuir a incapacidade relacionada à dor lombar dessensibilizando as preocupações e ansiedades e mudando as atitudes e crenças sobre a dor. Além de aumentar ou manter as funções musculoesqueléticas, com ganho de força, flexibilidade dorsal e de bônus melhora da aptidão cardiovascular (Gasibat et al., 2019). Em adição, Rios et al. (2015), afirmam que intervenções para dores crônicas apresentam um melhor resultado 
com a utilização de métodos para capacitar e educar os pacientes com relação a dor, e nesse contexto, além do exercício físico, a educação em dor diminui o caráter cíclico da dor crônica ao alterar a percepção do paciente sobre a dor, dano tecidual e funcionalidade (Malfliet et al., 2018).

Em relação aos gastos em saúde é perceptível que os pacientes apresentaram respostas que não diferiram ao longo do tempo. Posto isso, é importante destacar que a efetividade do condicionamento físico como estratégia de retorno ao trabalho e de redução de absenteísmo em pacientes com dor lombar crônica comparada à de tratamento convencional mostrou pequeno benefício em 12 meses (Schaafsma et al., 2013).

Uma das principais limitações desse estudo é o tamanho da amostra, que devido a perdas de seguimento, foi reduzida e a ausência de um grupo controle que não recebesse intervenção alguma. Uma das hipóteses é que esses dois fatores teriam auxiliado na comparação dos resultados, principalmente em relação aos desfechos psicossociais. Ademais, o tempo de tratamento parece não ter sido suficiente quando se considera a comparação entre os grupos, e o efeito da intervenção a longo prazo poderia ser avaliado com seguimento de pelo menos três meses. Sendo assim, estudos futuros são necessários para avaliar os efeitos a longo prazo da intervenção combinada.

\section{Conclusão}

Conclui-se que, um programa de educação em dor combinado com exercícios fisioterapêuticos é eficaz para melhora da funcionalidade física, dor, redução da incapacidade e cinesiofobia para o grupo lombalgia. Enquanto, para o grupo com sensibilização central houve melhora do nível de atividade física, mobilidade, dor e redução da incapacidade. E quanto aos gastos em saúde, percebe-se que não houve modificações ao longo do tempo, para internações e gastos com consultas médicas, visto o curto período de tempo avaliado.

Como perspectiva futura para esse trabalho há o melhoramento do protocolo de tratamento para que seja mais eficaz em pacientes com sensibilização central. Além disso, acredita-se que o acompanhamento a longo prazo desses pacientes servirá para a compressão do comportamento dos mesmos após a intervenção.

Por fim, esperamos que os resultados encontrados nesta pesquisa possam contribuir para a implantação de programas e protocolos de atendimento, para pacientes com dor lombar crônica, mais eficazes na rede SUS reduzindo assim as filas de esperas e os gastos em saúde.

\section{Agradecimentos}

Gostaríamos de agradecer o apoio logístico do Hospital Universitário de Sergipe e de seus funcionários, ao CNPQ pelo incentivo a pesquisa, ao Ministério da Saúde por meio do Programa Pesquisa para o SUS (PPSUS), que permitiu o financiamento do estudo e distribuição de bolsas de estudo e a todos os voluntários que disponibilizaram seu tempo para engrandecer a ciência.

\section{Referências}

Abreu, A. M. D., Faria, C. D. C. D. M., Cardoso, S. M. V., \& Teixeira-Salmela, L. F. (2008). Versão brasileira do fear avoidance beliefs questionnaire. Cadernos de Saúde Pública, 24, 615-623.

Airaksinen, O., Brox, J. I., Cedraschi, C., Hildebrandt, J., Klaber-Moffett, J., Kovacs, F., \& COST B13 Working Group on Guidelines for Chronic Low Back Pain. (2006). European guidelines for the management of chronic nonspecific low back pain. European spine journal, 15(Suppl 2), s192.

Almeida, V. C., Lima, V. B., Costa, K. M., Sandes, S. S., de Farias Neto, J. P., \& da Silva Junior, W. M. (2021). Do patients with low back pain and central sensitization have differences in physical fitness? Journal of Bodywork and Movement Therapies.

Aoyagi, K., He, J., Nicol, A. L., Clauw, D. J., Kluding, P. M., Jernigan, S., \& Sharma, N. K. (2019). A subgroup of chronic low back pain patients with central sensitization. The Clinical journal of pain, 35(11), 869 . 
Barbosa, J. E. S., dos Santos Almeida, L. P., de Oliveira, M. P., do Sacramento, M. D. S., Gomes, V. A., Petto, J., \& dos Santos, A. C. N. (2019). Influência do músculo diafragma no controle postural, na propriocepção e na dor lombar. Revista Brasileira de Fisiologia do Exercício, 18(4), $236-246$.

Caumo, W., Antunes, L. C., Elkfury, J. L., Herbstrith, E. G., Sipmann, R. B., Souza, A., \& Neblett, R. (2017). The Central Sensitization Inventory validated and adapted for a Brazilian population: psychometric properties and its relationship with brain-derived neurotrophic factor. Journal of pain research, 10, 2109.

Chiarotto, A., Terwee, C. B., \& Ostelo, R. W. (2016). Choosing the right outcome measurement instruments for patients with low back pain. Best practice \& research Clinical rheumatology, 30(6), 1003-1020.

Costa, L. D. C. M., Maher, C. G., McAuley, J. H., Hancock, M. J., Herbert, R. D., Refshauge, K. M., \& Henschke, N. (2009). Prognosis for patients with chronic low back pain: inception cohort study. Bmj, 339.

Costa, L. O. P., Maher, C. G., Latimer, J., Ferreira, P. H., Ferreira, M. L., Pozzi, G. C., \& Freitas, L. M. A. (2008). Clinimetric testing of three self-report outcome measures for low back pain patients in Brazil: which one is the best? Spine, 33(22), 2459-2463.

Estrela, C. (2018). Metodologia científica: ciência, ensino, pesquisa. Artes Médicas.

Foster, N. E., Anema, J. R., Cherkin, D., Chou, R., Cohen, S. P., Gross, D. P., \& Woolf, A. (2018). Prevention and treatment of low back pain: evidence, challenges, and promising directions. The Lancet, 391(10137), 2368-2383.

Foster, N. E., Thomas, E., Bishop, A., Dunn, K. M., \& Main, C. J. (2010). Distinctiveness of psychological obstacles to recovery in low back pain patients in primary care. $P A I N \circledast, 148(3), 398-406$.

Gasibat, Q., Mesrati, M. H., Musa, R. M., \& Zidan, A. A. (2019). Effective Recovery and Control of Chronic Low Back Pain by using Rehabilitation Exercises Therapy. Research Journal of Pharmacy and Technology, 12(9), 4313-4323.

Gore, M., Sadosky, A., Stacey, B. R., Tai, K. S., \& Leslie, D. (2012). The burden of chronic low back pain: clinical comorbidities, treatment patterns, and health care costs in usual care settings. Spine, 37(11), E668-E677.

Hoy, D., March, L., Brooks, P., Blyth, F., Woolf, A., Bain, C., \& Buchbinder, R. (2014). The global burden of low back pain: estimates from the Global Burden of Disease 2010 study. Annals of the rheumatic diseases, 73(6), 968-974.

Igwesi-Chidobe, C. N., Coker, B., Onwasigwe, C. N., Sorinola, I. O., \& Godfrey, E. L. (2017). Biopsychosocial factors associated with chronic low back pain disability in rural Nigeria: a population-based cross-sectional study. BMJ global health, 2(3), e000284.

Jensen, O. K., Nielsen, C. V., \& Stengaard-Pedersen, K. (2010). One-year prognosis in sick-listed low back pain patients with and without radiculopathy. Prognostic factors influencing pain and disability. The Spine Journal, 10(8), 659-675.

Kim, H., \& Lee, S. (2020). Effects of pain neuroscience education on kinesiophobia in patients with chronic pain: a systematic review and metaanalysis. Physical Therapy Rehabilitation Science, 9(4), 309-317.

Kolu, P., Tokola, K., Kankaanpää, M., \& Suni, J. (2017). Evaluation of the effects of physical activity, cardiorespiratory condition, and neuromuscular fitness on direct healthcare costs and sickness-related absence among nursing personnel with recurrent nonspecific low back pain. Spine, 42(11), 854-862.

Magnusson, S. P., Simonsen, E. B., Aagaard, P., Boesen, J., Johannsen, F., \& Kjaer, M. (1997). Determinants of musculoskeletal flexibility: viscoelastic properties, cross-sectional area, EMG and stretch tolerance. Scandinavian journal of medicine \& science in sports, 7(4), 195-202.

Malfliet, A., Kregel, J., Coppieters, I., De Pauw, R., Meeus, M., Roussel, N., \& Nijs, J. (2018). Effect of pain neuroscience education combined with cognition-targeted motor control training on chronic spinal pain: a randomized clinical trial. JAMA neurology, 75(7), 808-817.

Manning, K., Kauffman, B. Y., Rogers, A. H., Garey, L., \& Zvolensky, M. J. (2020). Fatigue severity and fatigue sensitivity: relations to anxiety, depression, pain catastrophizing, and pain severity among adults with severe fatigue and chronic low back pain. Behavioral medicine, 1-9.

Marques, J. S. R. (2020). Influência da dor lombar crônica inespecífica com ou sem sensibilização central nos aspectos biopsicossociais e capacidade funcional de idosos (Dissertação de Mestrado) Universidade Federal de São Carlos. São Carlos, SP.

Maughan, E. F., \& Lewis, J. S. (2010). Outcome measures in chronic low back pain. European Spine Journal, 19(9), $1484-1494$.

Meints, S. M., Mawla, I., Napadow, V., Kong, J., Gerber, J., Chan, S. T., \& Edwards, R. R. (2019). The relationship between catastrophizing and altered pain sensitivity in patients with chronic low back pain. Pain, 160(4), 833.

Miyamoto, G. C., Lin, C. W. C., Cabral, C. M. N., van Dongen, J. M., \& van Tulder, M. W. (2019). Cost-effectiveness of exercise therapy in the treatment of non-specific neck pain and low back pain: a systematic review with meta-analysis. British journal of sports medicine, 53(3), 172-181.

Moseley, G. L. (2005). Widespread brain activity during an abdominal task markedly reduced after pain physiology education: fMRI evaluation of a single patient with chronic low back pain. Australian Journal of Physiotherapy, 51(1), 49-52.

O’Neill, S., Manniche, C., Graven-Nielsen, T., \& Arendt-Nielsen, L. (2007). Generalized deep-tissue hyperalgesia in patients with chronic low-back pain. European journal of pain, 11(4), 415-420.

Pardo, G. B., Girbés, E. L., Roussel, N. A., Izquierdo, T. G., Penick, V. J., \& Martín, D. P. (2018). Pain neurophysiology education and therapeutic exercise for patients with chronic low back pain: a single-blind randomized controlled trial. Archives of physical medicine and rehabilitation, $99(2), 338-347$.

Rios, J. C. S., Leite, T. K. M., de Moura Pereira, M., Sousa, F. C., \& Safons, M. P. (2015). Effects of an educational self-care program of spine in elderly patients with chronic low back pain: a quasi-experimental study/Efeitos de um programa educacional de autocuidado de coluna em idosos com dor lombar cronica: um estudo quasi-experimental. Motricidade, 11(1), 53-64. 
Research, Society and Development, v. 10, n. 11, e404101119715, 2021

(CC BY 4.0) | ISSN 2525-3409 | DOI: http://dx.doi.org/10.33448/rsd-v10i11.19715

Ross, G. B., Sheahan, P. J., Mahoney, B., Gurd, B. J., Hodges, P. W., \& Graham, R. B. (2017). Pain catastrophizing moderates changes in spinal control in response to noxiously induced low back pain. Journal of biomechanics, 58, 64-70.

Schaafsma, F. G., Whelan, K., van der Beek, A. J., van der Es-Lambeek, L. C., Ojajärvi, A., \& Verbeek, J. H. (2013). Physical conditioning as part of a return to work strategy to reduce sickness absence for workers with back pain. Cochrane Database of Systematic Reviews, (8).

Sehn, F. C., Souza, I. C. C. D., Caumo, W., Torres, I. L. D. S., Vidor, L. P., \& Dall'Agnol, L. (2012). Validação da escala de pensamentos catastróficos para o português do Brasil. Revista HCPA. Porto Alegre.

Semedo, A. R. D. S. (2017). Modelos de sobrevivência para o estudo dos fatores de prognóstico até à ocorrência da resposta ao tratamento de fisioterapia em utentes com dor lombar crónica (Doctoral dissertation, Instituto Politécnico de Setúbal. Escola Superior de Saúde).

Silva, A. N., \& Martins, M. R. I. (2014). Dor, cinesiofobia e qualidade de vida de pacientes com dor lombar. Revista Dor, 15, 117-120.

Steffens, D., Maher, C. G., Pereira, L. S., Stevens, M. L., Oliveira, V. C., Chapple, M., \& Hancock, M. J. (2016). Prevention of low back pain: a systematic review and meta-analysis. JAMA internal medicine, 176(2), 199-208.

Tagliaferri, S. D., Miller, C. T., Owen, P. J., Mitchell, U. H., Brisby, H., Fitzgibbon, B., \& Belavy, D. L. (2020). Domains of chronic low back pain and assessing treatment effectiveness: a clinical perspective. Pain Practice, 20(2), 211-225.

Traeger, A. C., Moseley, G. L., Hübscher, M., Lee, H., Skinner, I. W., Nicholas, M. K., \& McAuley, J. H. (2014). Pain education to prevent chronic low back pain: a study protocol for a randomised controlled trial. BMJ open, 4(6), e005505.

Verkerk, K., Luijsterburg, P. A., Heymans, M. W., Ronchetti, I., Pool-Goudzwaard, A. L., Miedema, H. S., \& Koes, B. W. (2013). Prognosis and course of disability in patients with chronic nonspecific low back pain: a 5-and 12-month follow-up cohort study. Physical therapy, 93(12), $1603-1614$.

Walker, J., Sofaer, B., \& Holloway, I. (2006). The experience of chronic back pain: accounts of loss in those seeking help from pain clinics. European Journal of Pain, 10(3), 199-207.

Wong, J. J., Côté, P., Sutton, D. A., Randhawa, K., Yu, H., Varatharajan, S., \& Taylor-Vaisey, A. (2017). Clinical practice guidelines for the noninvasive management of low back pain: A systematic review by the Ontario Protocol for Traffic Injury Management (OPTIMa) Collaboration. European journal of pain, 21(2), 201-216.

Wood, L., \& Hendrick, P. A. (2019). A systematic review and meta-analysis of pain neuroscience education for chronic low back pain: Short-and long-term outcomes of pain and disability. European Journal of Pain, 23(2), 234-249.

Wu, A., March, L., Zheng, X., Huang, J., Wang, X., Zhao, J., \& Hoy, D. (2020). Global low back pain prevalence and years lived with disability from 1990 to 2017: estimates from the Global Burden of Disease Study 2017. Annals of translational medicine, 8(6).

Zanuto, E. A. C., Codogno, J. S., Christófaro, D. G. D., Vanderlei, L. C. M., Cardoso, J. R., \& Fernandes, R. A. (2015). Prevalence of low back pain and associated factors in adults from a middle-size Brazilian city. Ciencia \& saude coletiva, 20, 1575-1582. 